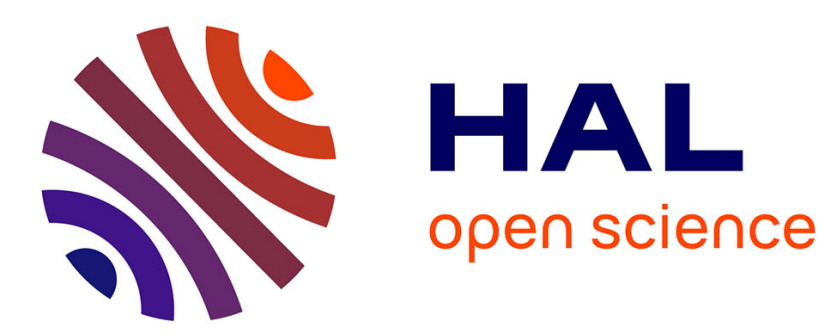

\title{
Observer design for Lipschitz systems with discrete-time measurements
}

\author{
Vincent Andrieu, Madiha Nadri
}

\section{To cite this version:}

Vincent Andrieu, Madiha Nadri. Observer design for Lipschitz systems with discrete-time measurements. 49th IEEE Conference on Decision and Conrtrol, Dec 2010, Atlanta, France. hal-00519361

\section{HAL Id: hal-00519361 \\ https://hal.science/hal-00519361}

Submitted on 20 Sep 2010

HAL is a multi-disciplinary open access archive for the deposit and dissemination of scientific research documents, whether they are published or not. The documents may come from teaching and research institutions in France or abroad, or from public or private research centers.
L'archive ouverte pluridisciplinaire HAL, est destinée au dépôt et à la diffusion de documents scientifiques de niveau recherche, publiés ou non, émanant des établissements d'enseignement et de recherche français ou étrangers, des laboratoires publics ou privés. 


\title{
Observer design for Lipschitz systems with discrete-time measurements
}

\author{
Vincent Andrieu and Madiha Nadri
}

\begin{abstract}
In this paper, the authors investigate the problem of designing an observer for Lipschitz nonlinear systems with discrete time measurements (continuous-discrete time systems). The result is based on reachability analysis to synthesize an upper approximation of a reachable set. When this approximation is given in terms of a convex combination of linear mappings, a sufficient condition is given in terms of linear matrix inequality which can be solved using LMI techniques. This approach seems to provide an efficient new tool to address the problem of observer design for a class of Lipschitz systems. An academic example is given to illustrates this point.
\end{abstract}

Keywords: nonlinear observer design, continuous discrete-time systems, reachability set, LMI.

\section{INTRODUCTION}

The problem under consideration in this paper is a state estimation problem for a class of Lipschitz continuous time systems with discrete time measurements. The use of continuous-discrete observers to estimate the state of Lipschitz nonlinear systems has already been investigated in the literature. It can be traced back to Jazwinski who introduced the continuous-discrete Kalman filter to solve a filtering problem for stochastic continuous-discrete time systems (see [5]). Inspired by this approach, the popular high-gain observer introduced in [4] has been adapted to the continuous-discrete context in [3]. In this work, the algorithm updates the estimate in two different ways: i) when no measurement is available, the estimate is obtained by integrating the model. ii) when a measurement occurs, the observer makes an impulsive correction of the estimate.

Note that in [3] the correction gain of this impulsive correction is obtained by integrating a continuous-discrete time Riccati equation. However, it has been shown in [8] that a constant gain can also be employed.

Since the seminal paper [3], this kind of continuous discrete time observers have also been employed on some other class of nonlinear systems in [9] and [1].

In all approaches mentioned above, the asymptotic convergence of the estimate to the state is obtained by dominating the Lipschitz nonlinearities with high-gain techniques. This can lead to restrictive design conditions on the sampling measurement time.

Recently, a new observer design methodology for Lipschitz nonlinear systems with continuous time measurements has

Vincent Andrieu and Madiha Nadri are with Université de Lyon, F-69622, Lyon, France; Université Lyon 1, Villeurbanne; CNRS, UMR 5007, LAGEP (Laboratoire d'Automatique et de GEnie des Procédés). 43 bd du 11 novembre, 69100 Villeurbanne, France https://sites.google.com/site/vincentandrieu/, nadrielagep.univ-lyon 1 . fr been introduced by Zemouche et. al. in [14]. In their approach, it is shown that the differential equation satisfied by the estimation error can be rewritten in the form of a linear parameter varying system (LPV). Hence, it is shown in [14] that the convergence to zero of the estimation error can be obtained by solving some specific linear matrix inequalities (see Section II for a brief summary of this approach).

The aim of this paper is to extend the approach presented in [14] to the discrete time measurement case. In the adopted strategy, the main problem is decomposed into two subproblems: i) The first one is concerned with the computation of an upper approximation of a reachable set for a bilinear system. This set characterizes the possible expansion of the estimation error between two measurements when the estimate is given by integrating the model.

ii) The second one is devoted to the construction of a correction term $K$ ensuring the convergence to zero of a quadratic error Lyapunov function. As in [14], this step is performed through LMI techniques.

It has to be noticed that a new result, which does not use high-gain techniques, has already been obtained in $[11]^{1}$. In this paper, the observer takes the form of a copy of the dynamic to which a correction term is added with hybrid dynamic: it is constant between two measurements and is updated at the time instant in which a new measurement is available. The observer gain is synthesized to guarantee that a particular error Lyapunov function (obtained from the work of [10]) is strictly decreasing along the trajectories. To obtain the observer gain some LMI conditions have to be satisfied. This approach provides an efficient way to solve the estimation problem. However, on some specific examples (see Section IV-B), the new LMI condition gives a solution when the approach presented in [11] seems to fail.

The rest of this paper is organized as follows : In Section II, after having defined the considered class of systems, some preliminary results and in particular the approach of [14] for continuous time measurements are recalled. An approach of observer design based on reachability set analysis is presented in Section III. Section IV, is devoted to the study of a particular class of systems: feedforward systems. In this Section, to illustrate the different step of the proposed approach, a numerical example is given.

\section{Preliminaries}

\section{A. The problem under consideration}

Without loss of generality, the class of nonlinear systems under consideration is described by the following differential

\footnotetext{
${ }^{1}$ The result presented in [11] addresses a more general problem since the sampling measurement time may be time varying.
} 
equation :

$$
\dot{x}(t)=A x(t)+\phi(x(t), u(t)),
$$

where the state $x$ is in $\mathbb{R}^{n}, u: \mathbb{R} \rightarrow \mathbb{R}^{p}$ is a known input, $A$ is a matrix in $\mathbb{R}^{n \times n}$ and $\phi: \mathbb{R}^{n} \times \mathbb{R}^{p} \rightarrow \mathbb{R}^{n}$ is a (uniformly in the input) globally Lipschitz function. In other words, the following assumption is considered:

Assumption 1: For each $(i, j)$ in $[1, n]^{2}$, there exist a positive real number $c_{i j}$ such that for all $(x, u)$ in $\mathbb{R}^{n} \times \mathbb{R}^{p}$ :

$$
\left|\frac{\partial \phi_{i}}{\partial x_{j}}(x, u)\right| \leq c_{i j} .
$$

The state $x$ of System (1) is accessible via discrete time measurements

$$
y_{k}=C x\left(t_{k}\right),
$$

where $\left(t_{k}\right)_{k \in \mathbb{N}}$ is a sequence of positive real numbers defined as: $t_{k+1}=t_{k}+\delta$ and $\delta$ is a positive real number representing the sampling measurement time.

The main objective of this work is to synthesize a global observer for system (1) which gives an estimate $\hat{x}$ which converges asymptotically to $x$ from the knowledge of the output $y_{k}$ given in (3).

Inspired by [3] and [8], the analysis is restricted to a specific class of continuous-discrete time observers defined by the following hybrid system ${ }^{2}$ :

$$
\left\{\begin{array}{l}
\dot{\hat{x}}(t)=A \hat{x}(t)+\phi(\hat{x}(t), u(t)), t \in\left[t_{k}, t_{k+1}\right), \\
\hat{x}\left(t_{k}\right)=\hat{x}\left(t_{k}^{-}\right)+K\left(y_{k}-C \hat{x}\left(t_{k}^{-}\right)\right),
\end{array}\right.
$$

where,

$$
\hat{x}\left(t_{k}^{-}\right)=\lim _{t \rightarrow t_{k} t<t_{k}} \hat{x}(t) .
$$

The estimation problem consists in determining a gain $K$ such that the estimation error $e(t)=x(t)-\hat{x}(t)$ converges asymptotically to zero. The proposed approach is based on an existing result obtained in [14] which is recalled in the next section.

\section{B. Approach of [14] in the continuous time case}

The approach published in [14] deals with the observer design for System (1) but the output is a continuous time function (measurements are continuously available) and given by:

$$
y(t)=C x(t), \forall t .
$$

Let $\mathcal{R}$ be the set of matrices in $\mathbb{R}^{n \times n}$ such that for all matrix $R=\left(R_{i j}\right)_{(i, j) \in[1, n]^{2}}$ in $\mathcal{R}$, components $R_{i j}$ takes two values $A_{i j}+c_{i j}$ or $A_{i j}-c_{i j}$

Note that this set of matrices is composed of $2^{\rho}$ elements where $\rho$ is the number of $c_{i j} \neq 0$.

In [14], one of the results they obtain can be summarized by the following theorem.

Theorem 1 (Continuous time measurement case, [14]):

Assume assumption 1 is satisfied for System (1). If there exist a symmetric positive definite (SPD) matrix $P$ in

\footnotetext{
${ }^{2}(A, C)$ is assumed to be observable
}

$\mathbb{R}^{n \times n}$ and a vector $L$ in $\mathbb{R}^{n}$ such that the following matrix inequalities hold:

$$
R^{\prime} P+P R-C^{\prime} L-L^{\prime} C<0, \forall R \in \mathcal{R},
$$

then the system

$$
\dot{\hat{x}}(t)=A \hat{x}(t)+\phi(\hat{x}(t), u(t))+P^{-1} L(y(t)-C \hat{x}(t),
$$

is an asymptotic observer for system (1) where $y$ is the continuous time measure given by (6). i.e.

$$
\lim _{t \rightarrow+\infty}|\hat{x}(t)-x(t)|=0 .
$$

The present paper, gives an extension of this result to the case where the measure is a discrete time function (3).

In the case of feedforward systems, it is shown in Section IV that if the sampling measurement time period goes to zero, the result of Theorem 1 ([14]) is obtained.

\section{AN APPROACH BASED ON REACHABLE SET COMPUTATION}

\section{A. The new approach}

The approach of observer design proposed in this work, is based on a reachable set computation. To develop this approach, the following controlled bilinear system is considered:

$$
\dot{e}(t)=A e(t)+U(t) e(t),
$$

with state $e$ in $\mathbb{R}^{n}$ and where $U$ in $\mathbb{R}^{n \times n}$ is a time varying matrix, whose elements,

$$
U(t)=\left(u_{i j}(t)\right) \quad, 1 \leq i \leq n \quad, \quad 1 \leq j \leq n,
$$

are some control inputs. Given system (8), and a positive real number $\delta$, the set valued map $e \in \mathbb{R}^{n} \mapsto \mathcal{U}_{\delta}(e) \subset \mathbb{R}^{n}$, which gives the reachable set at time $\delta$ with the control constraint $\left|u_{i j}\right| \leq c_{i j}$ can be defined. More precisely, for each $e_{0}$ in $\mathbb{R}^{n}$ and for each $e_{1}$ in $\mathcal{U}_{\delta}\left(e_{0}\right)$, there exists a function $t \mapsto U(t)$ such that for all $t$ in $[0, \delta]$,

$$
\left|u_{i j}(t)\right| \leq c_{i j} \quad, \quad 1 \leq i \leq n \quad, \quad 1 \leq j \leq n,
$$

such that the solution $e(t)$ of system (8) starting from $e_{0}$ with the control $u_{i j}(\cdot)$ satisfies $e(\delta)=e_{1}$.

Using this set valued map $\mathcal{U}_{\delta}$, a sufficient condition based on a linear matrix inequality which enables to design a continuous-discrete time observer (like (4)) for system (1)(3) and to guarantee its global convergence can be given.

Theorem 2: Assume assumption 1 is satisfied for System (1). Given $\delta$, the sampling measurement time, if there exists $\ell$ matrix functions $\left(t \mapsto M_{i}(t)\right)_{i=1, \ldots, \ell}$ mapping $\mathbb{R}_{+}$into $\mathbb{R}^{n \times n}$, a positive definite matrix $P$ in $\mathbb{R}^{n \times n}$ and a vector $W$ in $\mathbb{R}^{n}$ such that ${ }^{3}$ :

$$
\mathcal{U}_{\delta}(e) \subseteq \operatorname{Conv}_{i=\{1, \ldots, \ell\}}\left\{M_{i}(\delta) e\right\} \quad, \quad \forall e \in \mathbb{R}^{n}
$$

and such that the following matrix inequalities hold ${ }^{4}$,

$$
\left[\begin{array}{cc}
P & \star \\
(P+W C) M_{i}(\delta) & P
\end{array}\right]>0, \forall i \in[1, \ell],
$$

\footnotetext{
${ }^{3}$ The notation Conv denotes the convex closure given a set of vectors.
} ${ }^{4} \star$ represents the symmetric form of matrix; i.e., $\star=M_{i}^{\prime}(\delta)\left(P+C^{\prime} W^{\prime}\right)$ 
then the observer (4) with $K=P^{-1} W$ asymptotically estimates the state of the system (1).

Proof : Along the trajectories of the system (1) using the observer given in (4) the estimation error $e=\hat{x}-x$ satisfies

$$
\left\{\begin{array}{l}
\dot{e}(t)=A e(t)+\Delta \phi(\hat{x}(t), u(t), e(t)), \\
t \in[k \delta,(k+1) \delta) ; \\
e(k \delta)=\left(I_{n}-K C\right) e\left(k \delta^{-}\right),
\end{array}\right.
$$

where the notation (5) is used and where $I_{n}$ is the identity matrix of order $n$ and $\Delta \phi: \mathbb{R}^{n} \times \mathbb{R}^{n} \times \mathbb{R}^{p} \rightarrow \mathbb{R}^{n}$ is the continuous function defined as

$$
\Delta \phi(\hat{x}, u, e)=\phi(\hat{x}, u)-\phi(\hat{x}-e, u) .
$$

The mean value theorem yields the existence of $n$ functions $z_{i}: \mathbb{R}^{2 n} \rightarrow \mathbb{R}^{n}, i=1, \ldots, n$ such that the components of the function $\Delta \phi$ satisfy:

$$
\Delta \phi_{i}(\hat{x}, u, e)=\frac{\partial \phi_{i}}{\partial x}\left(z_{i}(\hat{x}, e), u\right) e, \quad i=1, \ldots, n
$$

Hence, for $t$ in $[k \delta,(k+1) \delta)$, the error is a solution of system (8) with "control" matrix $U(t)$, the components of which are defined as $u_{i j}(t)=\frac{\partial \phi_{i}}{\partial x_{i}}\left(z_{i}(\hat{x}(t), e(t)), u(t)\right)$.

On the other hand, with assumption 1, the global Lipschitz condition (2) yields that the "constraint" (9) is satisfied. Consequently, with the definition of the set valued function $\mathcal{U}_{\delta}$ it yields: $e\left(t_{k+1}^{-}\right) \in \mathcal{U}_{\delta}\left(e\left(t_{k}\right)\right)$.

This, with the inclusion (10) establishes that:

$$
e\left(t_{k+1}^{-}\right) \in \operatorname{Conv}_{i=\{1, \ldots, \ell\}}\left\{M_{i} e\left(t_{k}\right)\right\} .
$$

With the discrete dynamics of the error in (12), it yields:

$$
e\left(t_{k+1}\right) \in \operatorname{Conv}_{i=\{1, \ldots, \ell\}}\left\{(I+K C) M_{i} e\left(t_{k}\right)\right\} .
$$

In other word, there exist $\left(\alpha_{1}, \ldots, \alpha_{\ell}\right)$, with $\sum_{i=1}^{\ell} \alpha_{i}=1$ and $\alpha_{i} \geq 0$ such that $e\left(t_{k+1}\right)=\sum_{i=1}^{\ell} \alpha_{i}(I+K C) M_{i} e\left(t_{k}\right)$. Consider the $C^{1}$ quadratic function $V: e \mapsto e^{\prime} P e$. The evaluation of this function at $e=e\left(t_{k+1}\right)$, satisfies:

$$
e\left(t_{k+1}\right)^{\prime} P e\left(t_{k+1}\right)=\sum_{i=1}^{\ell} \sum_{j=1}^{\ell} \alpha_{i} \alpha_{j} e\left(t_{k}\right)^{\prime} N_{i}^{\prime} P N_{j} e\left(t_{k}\right)
$$

with $N_{j}=(I+K C) M_{j}$. The Cauchy-Schwarz inequality, leads to:

$$
\begin{aligned}
e\left(t_{k+1}\right)^{\prime} & P e\left(t_{k+1}\right) \\
\leq & \frac{1}{2} \sum_{i=1}^{\ell} \sum_{j=1}^{\ell} \alpha_{i} \alpha_{j} e\left(t_{k}\right)^{\prime}\left[N_{i}^{\prime} P N_{i}+N_{j}^{\prime} P N_{j}\right] e\left(t_{k}\right) .
\end{aligned}
$$

Hence, since $\sum_{i=1}^{\ell} \alpha_{i}=1$, it yields,

$$
\begin{aligned}
e\left(t_{k+1}\right)^{\prime} & P e\left(t_{k+1}\right) \\
\leq & \sum_{i=1}^{\ell} \alpha_{i} e\left(t_{k}\right)^{\prime} M_{i}^{\prime}(I+K C)^{\prime} P(I+K C) M_{i} e\left(t_{k}\right) .
\end{aligned}
$$

On the other hand, with equation (11) and the Schur Complement it yields that

$$
M_{i}^{\prime}(I+K C)^{\prime} P(I+K C) M_{i}-P<0, i=1, \ldots, \ell .
$$

Hence,

$$
M_{i}^{\prime}(I+K C)^{\prime} P(I+K C) M_{i} \leq\left(1-\kappa_{i}\right) P, i=1, \ldots, \ell
$$

where $0<\kappa_{i}<1$ is the positive real number defined as

$$
\kappa_{i}=\frac{\lambda_{\min }\left(P-M_{i}^{\prime}(I+K C)^{\prime} P(I+K C) M_{i}\right)}{\lambda_{\max }(P)},
$$

and $\lambda_{\max }(),. \lambda_{\min }($.$) are, respectively, the largest and the$ smallest eigenvalue. Consequently, this implies that,

$$
e\left(t_{k+1}\right)^{\prime} P e\left(t_{k+1}\right) \leq\left(1-\kappa_{m}\right) \sum_{i=1}^{\ell} \alpha_{i} e\left(t_{k}\right)^{\prime} P e\left(t_{k}\right) .
$$

where $\kappa_{m}=\min \left\{\kappa_{i}, i=\{1, \ldots, \ell\}\right\}$. Hence,

$$
V\left(e\left(t_{k}\right)\right) \leq\left(1-\kappa_{m}\right)^{k} V(e(0)) \text {. }
$$

For all $t \in\left[t_{k}, t_{k+1}\right)$, according to (12), it yields that $\overparen{V(e(t))}=e(t)^{\prime}\left(P A+A^{\prime} P\right) e(t)$

$$
+2 e(t)^{\prime} P \Delta \phi(\hat{x}(t), u(t), e(t)) .
$$

From (13), it follows that,

$$
\begin{aligned}
\overparen{V(e(t))}=e(t)^{\prime} & \left(P A+A^{\prime} P\right) e(t) \\
& +2 e(t)^{\prime} P \sum_{i=1}^{n} \frac{\partial \phi_{i}}{\partial x}\left(z_{i}(\hat{x}(t), e(t)), u(t)\right) e(t) .
\end{aligned}
$$

Assumption 1 implies that,

$$
\sum_{i=1}^{n} \frac{\partial \phi_{i}}{\partial x}\left(z_{i}(\hat{x}, e), u\right) e \in \operatorname{Conv}_{\{R \in \mathcal{R}\}}\{R e\} .
$$

Thus $\overparen{V(e(t))} \leq \eta V(e(t)), \forall t \in\left[t_{k}, t_{k+1}\right)$, where $\eta$ is the positive real number defined as

$$
\eta=\frac{\max _{R \in \mathcal{R}}\left\{\lambda_{\max }\left(P[A+R]+[A+R]^{\prime} P\right), 0\right\}}{\lambda_{\min }(P)} .
$$

Using (14), the following holds

$$
V(e(t)) \leq\left(1-\kappa_{m}\right)^{k} \exp (\eta \delta) V(e(0)), \forall t \in\left[t_{k}, t_{k+1}\right) .
$$

Since $1>\kappa_{m}>0$, the function $V(e(t))$ goes to zero as time goes to infinity. The function $V(e)$ being proper and definite positive, this implies that $e(t)$ converges to zero and finishes the proof.

\section{B. Discussion on assumptions}

The first step of the proposed approach is the computation of a reachable set for controlled bilinear systems. Reachability analysis has received numerous attentions in the literature; for instance, In [2], the author analyzes the geometry of the reachable set of bilinear systems. In [13], the author gave sufficient conditions guaranteeing that the reachable set of a bilinear controllable system is convex. If some results on the characterization of the reachable set are now available for low dimension systems (see for instance the recent result in [7]) its characterization is still an open problem for high dimension systems.

However, the novelty of the studied problematic is that the exact computation of this set is not needed but only an upper approximation in terms of the matrix functions $\left(t \mapsto M_{i}(t)\right)_{i=1, \ldots, \ell}$ as expressed in (10). As it will be seen in 
section IV, for feedfoward systems, an upper approximation can be explicitly given, which may solve the estimation problem.

Given the matrix functions $\left\{t \mapsto M_{i}(t)\right\}_{i=1, \ldots, \ell}$, the second step of the design is to solve a linear matrix inequality. In fact, it can be shown that if Zemouche et. al.'s approach, given in Theorem 1, applies for the continuous time measurement case, then for small sampling measurement time, the proposed approach can be applied provided the matrix functions $M_{i}$ satisfy some local properties. Indeed, the link between the two matrix inequalities (7) and (11) can be expressed as follows.

Proposition 1: Assume there exist $P$ and $L$ satisfying the matrix inequality (7) for a given set of matrix $\mathcal{R}$. If the set of matrix functions $\left\{t \mapsto M_{i}(t)\right\}_{i=1, \ldots, \ell}$ is such that:

1) for all $i, M_{i}(t)$ is a $C^{1}$ function such that $M_{i}(0)=I_{n}$,

2) it holds

$$
\left\{\frac{d M_{i}}{d t}(0)\right\}_{i=1, \ldots, \ell} \subseteq \mathcal{R},
$$

then for all sufficiently small $\delta$ the matrix inequalities (11) are satisfied with the same $P$ and with $W=\delta L$.

The proof of this result has been removed due to space limitation. Consequently, from Proposition 1, it follows that a good upper approximation of the reachable set $\mathcal{U}_{\delta}$ in terms of the matrix functions $M_{i}(\delta)$ should be those which satisfy (15). As it will be seen in the next section, this is indeed the case when feedforward systems are considered.

\section{APPLICATION TO FEEDFORWARD SYSTEMS}

\section{A. Construction of the $M_{i}$}

The approach presented in the previous Section can be applied when considering a specific class of Lipschitz nonlinear systems. Indeed, when the matrix $A$ and the function $\phi$ have an upper triangular structure, computing the matrix $M_{i}$ involved in the procedure, can be made explicitly.

The assumption made on the matrix $A$ and the function $\phi$ can be summarized as follows:

Assumption 2: $A$ is a matrix in $\mathbb{R}^{n \times n}$ such that

$$
A(i, j)=a_{i j}=0 \quad, \quad \forall 1 \leq j<i \leq n .
$$

Moreover, the function $\phi$ involved in the definition of system (1) is a smooth function which satisfies an upper triangular global Lipschitz condition. In other words, the positive real numbers $c_{i j}$ satisfy:

$$
c_{i j}=0 \quad, \quad \forall 1 \leq j<i \leq n .
$$

With assumption 2, the following result can be obtained.

Theorem 3 (Observer design for feedforward systems):

Consider System (8) with the control constraints (9). With assumption 2, there exists a positive integer $\ell$ and a set of $C^{1}$ matrix functions $\left(\delta \mapsto M_{i}(\delta)\right)_{1 \leq i \leq \ell}$ taking values in $\mathbb{R}^{n \times n}$ such that for each $\delta>0$ the inclusion (10) is satisfied. Moreover, for each $i$ in $[1, \ell], \frac{d M_{i}(0)}{d t} \subseteq \mathcal{R}$.

Proof : The proof of this result is based on the use of an iterative procedure described by this lemma, the proof of which is given in appendix.
Lemma 1 (Iterative design of the $\left(M_{i}\right)$ 's): Consider the following controlled system with state $(w, z)$ in $\mathbb{R} \times \mathbb{R}^{n_{z}}$ defined for all positive time $t$ by :

$$
\begin{aligned}
\dot{w}(t) & =v_{w}(t) w(t)+V(t) z(t), w(0)=w_{0} \\
z(t) & \in \mathrm{Conv}_{i=1, \ldots, \ell_{z}}\left\{M_{z, i}(t) z_{0}\right\}, z(0)=z_{0}
\end{aligned}
$$

where $v_{w}$ in $\mathbb{R}$ and $V=\left(v_{1}, \ldots, v_{n_{z}}\right)$ in $\mathbb{R}^{n_{z}}$ are the control inputs satisfying $v_{w, \min } \leq v_{w} \leq v_{w, \max }, v_{j, \min } \leq v_{j} \leq$ $v_{j, \max }$ for $j$ in $\left[1, n_{z}\right]$ and where $\ell_{z}$ is a positive integer and $\left(t \mapsto M_{z, i}(t)\right)_{1 \leq i \leq \ell_{z}}$ are $C^{1}$ matrix functions mapping $\mathbb{R}_{+}$ into $\mathbb{R}^{n_{z} \times n_{z}}$ such that $M_{z, i}(0)=I_{n_{z}}$. Then, there exist a positive integer $\ell_{w}$ and a set of $C^{1}$ matrix functions $(t \mapsto$ $\left.M_{w, i}(t)\right)_{1 \leq i \leq \ell_{w}}$ taking value in $\mathbb{R}^{n_{z}+1}$ such that,

1) for all positive time $t$,

$$
\left[\begin{array}{c}
w(t) \\
z(t)
\end{array}\right] \in \mathrm{Conv}_{i=1, \ldots, \ell}\left\{M_{w, i}(t)\left[\begin{array}{c}
w_{0} \\
z_{0}
\end{array}\right]\right\}
$$

2) for all $i$ in $\left[1, \ell_{w}\right], M_{w, i}(0)=I_{n_{z}+1}$.

3) for all $i$ in $\left[1, \ell_{w}\right]$, there exists a real number $\Lambda_{i}$, a vector $\Upsilon_{i}$ in $\mathbb{R}^{n_{z}}$ and an integer $k_{i}$ in $\left[1, \ell_{z}\right]$ such that

$$
\frac{d M_{w, i}}{d t}(0)=\left[\begin{array}{cc}
\Lambda_{i} & \Upsilon_{i} \\
0 & \frac{d M_{z, k}}{d t}(0)
\end{array}\right]
$$

where $\Lambda_{i} \in\left\{v_{w, \min }, v_{w, \max }\right\}$ and,

$$
\Upsilon_{i, j} \in\left\{v_{j, \min }, v_{j, \max }\right\}, j \in\left[1, n_{z}\right] .
$$

Step 1: In the first step of the iterative procedure, the dynamics of the last component of the error (i.e. $e_{n}$ ) in System (8) is considered. With assumption 2, this component satisfies:

$$
\dot{e}_{n}=\left[a_{n n}+u_{n n}\right] e_{n}
$$

with the control constraint $\left|u_{n n}\right| \leq c_{n n}$. The solutions of this system are given as:

$$
e_{n}(t)=\exp \left(\int_{0}^{t}\left(a_{n n}+u_{n n}(s)\right) d s\right) e_{n}(0) .
$$

This implies that:

$$
e_{n}(t) \in \operatorname{Conv}\left\{M_{n, 1} e_{n}(0), M_{n, 2} e_{n}(0)\right\}
$$

where,

$$
\begin{aligned}
& M_{n, 1}(t)=\exp \left(\left[a_{n n}-c_{n n}\right] t\right) \\
& M_{n, 2}(t)=\exp \left(\left[a_{n n}+c_{n n}\right] t\right) .
\end{aligned}
$$

Note that,

$$
\frac{d M_{n, 1}}{d t}(0)=a_{n n}-c_{n n}, \frac{d M_{n, 2}}{d t}(0)=a_{n n}+c_{n n} .
$$

Step j: Then iteratively Lemma 1 can be applied to get the result. Indeed, at step $j$ the dynamics of $e_{r}$ where $r=n-$ $j+1$ in System (8) is considered. With assumption 2, this one is given as

$$
\dot{e}_{r}=\left(a_{r, r}+u_{r, r}\right) e_{r}+\sum_{q=r+1}^{n}\left(a_{r, q}+u_{r, q}\right) e_{q} .
$$


It is assumed also that the previous step gives a set of $\ell_{r+1}$ matrix functions $\left(s \mapsto M_{r+1, i}(s)\right)_{1 \leq i \leq \ell_{r+1}}$ taking values in $\mathbb{R}^{(j-1) \times(j-1)}$ such that

1) the $j-1$ last components of the error vector (i.e. $\left.E_{r+1}=\left(e_{r+1}, \ldots, e_{n}\right)\right)$ satisfies:

$$
E_{r+1}(t) \in \operatorname{Conv}\left\{M_{r+1, i}(t) E_{r+1}(0)\right\} .
$$

2) for all $i$ in $\left[1, \ell_{r+1}\right], M_{r+1, i}(0)=I_{i-1}$,

3) for all $i$ in $\left[1, \ell_{r+1}\right]$, all $(p, q)$ in $[r+1, n]^{2}$,

$$
\left(\frac{d M_{r+1, i}}{d t}(0)\right)_{p-r, q-r} \in\left\{A_{p q}+c_{p q}, A_{p q}-c_{p q}\right\}
$$

Note that (19) and (20) give a system in the form of the one introduced in (16) of Lemma 1 with

$$
v_{w}=a_{r, r}+u_{r, r}, v_{j}=a_{r, j}+u_{r, j}, z=E_{r+1} .
$$

Consequently, applying Lemma 1, it yields the existence of a positive integer $\ell_{r}$ and a set of matrix functions $(t \mapsto$ $\left.M_{w, j}(t)\right)_{1 \leq i \leq \ell_{r}}$ taking values in $\mathbb{R}^{j \times j}$ such that

1) the $j$ last components of the error vector $E_{r}=$ $\left(e_{r}, \ldots, e_{n}\right)$ satisfies for all positive time $t$,

$$
E_{r}(t) \in \operatorname{Conv}\left\{M_{r, j}(t) E_{r}(0)\right\} .
$$

2) for all $i$ in $\left[1, \ell_{r}\right], M_{r, i}(0)=I_{j}$,

3) Using the structure of the matrix functions in (18) leads to, for all $i$ in $\left[1, \ell_{r}\right]$, all $(p, q)$ in $[r, n]^{2}$,

$$
\left(\frac{d M_{r, j}}{d t}(0)\right)_{p-r+1, q-r+1} \in\left\{A_{p q}+c_{p q}, A_{p q}-c_{p q}\right\}
$$

Consequently, at the last step of this iterative design, the result is obtained.

\section{B. Numerical Example}

We consider System (1) in the simple case where it is a second order system (i.e. $n=2$ ) and when the function $\phi$ is such that:

$$
\phi\left(x_{1}, x_{2}\right)=\left[\begin{array}{l}
\phi_{1}\left(x_{1}\right) \\
\phi_{2}\left(x_{2}\right)
\end{array}\right],
$$

where $\left|\phi_{1}^{\prime}\left(x_{1}\right)\right| \leq c_{L} \quad, \quad\left|\phi_{2}^{\prime}\left(x_{2}\right)\right| \leq c_{L}$. and where $A$ is defined as: $A=\left[\begin{array}{ll}0 & 1 \\ 0 & 0\end{array}\right]$.

Note that this system satisfies assumption 1 and 2 with $c_{11}=$ $c_{22}=c_{L}$. Now, consider the controlled error system (8) which in this case is simply: $\dot{e}_{1}=e_{2}+u_{11} e_{1}, \quad \dot{e}_{2}=u_{22} e_{2}$. With Theorem 3 (and more precisely Lemma 1) it's possible to construct a set of matrices $M_{i}(\delta)$ such that the mapping giving the reachable set at time $\delta, e \mapsto \mathcal{U}_{\delta}(e)$ satisfies (10). This set of matrices is given as the 12 matrix functions

$$
M_{i}(\delta)=\left[\begin{array}{cc}
a_{i} & b_{i} \\
0 & d_{i}
\end{array}\right]
$$

where

$$
\begin{aligned}
& a_{i}(\delta) \in\left\{\exp \left(-c_{L} \delta\right), \exp \left(c_{L} \delta\right)\right\} \\
& b_{i}(\delta) \in\left\{\delta \exp \left(\delta c_{L}\right), \frac{\exp \left(\delta c_{L}\right)-\exp \left(-\delta c_{L}\right)}{2 c_{L}}, \delta \exp \left(-\delta c_{L}\right)\right\} \\
& d_{i}(\delta) \in\left\{\exp \left(-c_{L} \delta\right), \exp \left(c_{L} \delta\right)\right\}
\end{aligned}
$$

Note that the LMI test may be simplified since

$$
\delta \exp \left(\delta c_{L}\right) \leq \frac{\exp \left(\delta c_{L}\right)-\exp \left(-\delta c_{L}\right)}{2 c_{L}} \leq \delta \exp \left(-\delta c_{L}\right)
$$

and then consider $b_{i} \in\left\{\delta \exp \left(\delta c_{L}\right), \delta \exp \left(-\delta c_{L}\right)\right\}$.

Consequently, the number of matrices is reduced from 12 to 8 matrices.

Employing the Yalmip package ([6]) in Matlab in combination with the solver Sedumi ([12]), it can be checked that the LMI (11) is satisfied when $c_{L}=1$ and $\delta \leq 0.4$. The observer gain obtained is: $K=[-1.0017,-2.0478]^{\prime}$.

Note that given $c_{L}=1$, the measurement stepsize $\delta$ seems (numerically) to be maximized at the value 0.4 for this approach.

To compare with the result obtained in [11], the simpler case in which $\phi_{1}\left(x_{1}\right)=0$ in order to fit in the context of both approaches is considered. Hence, in this case, $c_{11}=0$. Applying the previous procedure and again, employing the same software for $\delta=0.5$, it can be checked that the LMI (11) is satisfied ${ }^{5}$ when $c_{L}=1$. Note, however, that the matrix inequality conditions of [11] with the same data failed to be solved. This fact shows the interest of the proposed method to complement existing results.

\section{CONCLUSION}

In this paper, the problem of designing an observer for nonlinear systems with discrete time measurements and globally Lipschitz nonlinearities is addressed. A solution based on the synthesis of an upper approximation of a reachable set have been presented. When this approximation is given in terms of a convex combination of linear mappings, a sufficient condition of the global convergence of the proposed observer is obtained in terms of a linear matrix inequality. The good performances obtained on an illustration example demonstrates that the proposed approach is an efficient tool.

\section{REFERENCES}

[1] T. Ahmed Ali, R. Postoyan, and F. Lamnabhi-Lagarrigue. Continuousdiscrete adaptive observers for state affine systems. Automatica, 45(12).

[2] R. W. Brockett. On the reachable set for bilinear system. 111:54-63, 1975.

[3] F. Deza, E. Busvelle, JP Gauthier, and D. Rakotopara. High gain estimation for nonlinear systems. Systems \& control letters, 18(4):295299, 1992.

[4] J. P. Gauthier, H. Hammouri, and S. Othman. A simple observer for nonlinear systems applications to bioreactors. IEEE Transactions on Automatic Control, 37(6):875-880, 1992.

[5] A.H. Jazwinski. Stochastic processes and filtering theory. Mathematics in Science and Engineering, 1970.

${ }^{5}$ The Matlab files can be downloaded from 
[6] J. Löfberg. Yalmip : A toolbox for modeling and optimization in MATLAB. In Proc. of the CACSD Conference, Taipei, Taiwan, 2004.

[7] M. Margaliot and M.S. Branicky. Nice reachability for planar bilinear control systems with applications to planar linear switched systems. IEEE Transactions on Automatic Control, 54(6):1430-1435, 2009.

[8] M. Nadri and H. Hammouri. Constant gain observer for continuousdiscrete time uniformly observable systems. Submitted to System \& Control letters, 2010.

[9] M. Nadri, H. Hammouri, and C. Astorga. Observer design for continuous discrete-time state affine systems up to output injection. European Journal of Control, 10(3):252-262, 2004.

[10] P. Naghshtabrizi, J. Hespanha, and A. Teel. Exponential stability of impulsive systems with application to uncertain sampled-data systems. Systems \& Control Letters, 57(5):378-385, 2008.

[11] T. Raff, M. Kogel, and F. Allgower. Observer with sample-and-hold updating for Lipschitz nonlinear systems with nonuniformly sampled measurements. In Proc. of American Control Conference, pages 52545257, 2008.

[12] J.F. Sturm. Using SeDuMi 1.02, a MATLAB toolbox for optimization over symmetric cones. Optimization Methods and Software, 1112:625-653, 1999. http://sedumi.mcmaster.ca/.

[13] M. V. Topunov. The convexity of the reachable set for a bilinear controllable system. Journal of Applied Mathematics and Mechanics, 67(5):665-670, 2003.

[14] A. Zemouche, M. Boutayeb, and G.I. Bara. Observers for a class of Lipschitz systems with extension to H8 performance analysis. Systems \& Control Letters, 57(1):18-27, 2008.

\section{APPENDIX}

\section{A. Proof of Lemma 1}

The solution of the system (16) satisfies:

$$
\begin{aligned}
& w(t)=\exp \left(\int_{0}^{t} v_{w}(s) d s\right) w(0)+ \\
& \int_{0}^{t} \exp \left(\int_{s}^{t} v_{w}(r) d r\right) V(s) z(s) d s,
\end{aligned}
$$

where $V(s)=\left[v_{1}, \ldots, v_{n_{z}}\right] .\left(\bar{V}_{k}\right)$ is the $2^{n_{z}}$ matrix column in $\mathbb{R}^{n_{z}}$ such that for each $k$, the $j^{\text {th }}$ component denoted $\bar{V}_{k, j}$ satisfies, $\bar{V}_{k, j} \in\left\{v_{j, \min }, v_{j, \max }\right\}$

$\left((s, t) \mapsto \gamma_{r}(s, t)\right)_{1 \leq r \leq 4^{n_{z}}}$ denotes the $4^{n_{z}}$ vector functions taking value in $\mathbb{R}^{n_{z}}$ such that for each $r$ in $\left\{1, \ldots, 4^{n_{z}}\right\}$, the $j^{\text {th }}$ component of $\gamma_{r}$ (denoted $\left.\left(\gamma_{r}(s, t)\right)_{j}\right)$ satisfies,

$$
\begin{aligned}
& \left(\gamma_{r}(s, t)\right)_{j} \in\{ \\
& \min _{i \in\left[1, \ell_{z}\right], k \in\left[1,2^{n_{z}}\right]}\left\{\exp \left((t-s) v_{w, \min }\right)\left(\bar{V}_{k}(s) M_{z, i}(s)\right)_{j}\right\}, \\
& \max _{i \in\left[1, \ell_{z}\right], k \in\left[1,2^{n_{z}}\right]}\left\{\exp \left((t-s) v_{w, \min }\right)\left(\bar{V}_{k}(s) M_{z, i}(s)\right)_{j}\right\} \text {, } \\
& \min _{i \in\left[1, \ell_{z}\right], k \in\left[1,2^{n z}\right]}\left\{\exp \left((t-s) v_{w, \max }\right)\left(\bar{V}_{k}(s) M_{z, i}(s)\right)_{j}\right\} \text {, } \\
& \max _{i \in\left[1, \ell_{z}\right], k \in\left[1,2^{n_{z}}\right]}\left\{\exp \left((t-s) v_{w, \max }\right)\left(\bar{V}_{k}(s) M_{z, i}(s)\right)_{j}\right\} \\
& \} \text {. }
\end{aligned}
$$

Note that for each $j$ in $\left\{1, \ldots, n_{z}\right\}$, it holds:

$$
\begin{aligned}
& \left(V(s) M_{z, i}(s)\right)_{j}\left(z_{0}\right)_{j} \leq \\
& \left\{\begin{array}{r}
\left(\max _{i \in\left[1, \ell_{z}\right], k \in\left[1,2^{n z}\right]}\left\{\left(\bar{V}_{k}(s) M_{z, i}(s)\right)_{r}\right\}\right)\left(z_{0}\right)_{j} \\
\text { if }\left(z_{0}\right)_{j} \geq 0, \\
\left(\min _{i \in\left[1, \ell_{z}\right], k \in\left[1,2^{n_{z}}\right]}\left\{\left(\bar{V}_{k}(s) M_{z, i}(s)\right)_{r}\right\}\right)\left(z_{0}\right)_{j} \\
\text { if }\left(z_{0}\right)_{j} \leq 0
\end{array}\right.
\end{aligned}
$$

This implies, that for all $z_{0}$ in $\mathbb{R}^{n_{z}}$ there exists $r_{1}$ in $\left\{1, \ldots, 4^{n_{z}}\right\}$ such that for all $i$ in $\left\{1, \ldots, \ell_{z}\right\}$, and all $(s, t)$

$$
\exp \left(\int_{s}^{t} v_{w}(r) d r\right) V(s) M_{z, i}(s) z_{0} \leq \gamma_{r_{1}}(s, t) z_{0} .
$$

Hence,

$$
\begin{aligned}
\int_{0}^{t} \exp \left(\int_{s}^{t} v_{w}(r) d r\right) V(s) M_{z, i}(s) z_{0} & \\
& \leq \int_{0}^{t} \gamma_{r_{1}}(s, t) z_{0} d s .
\end{aligned}
$$

Similarly, for all $z_{0}$ in $\mathbb{R}^{n_{z}}$ there exists $r_{2}$ in $\left\{1, \ldots, 4^{n_{z}}\right\}$ such that for all $i$ in $\left\{1, \ldots, \ell_{z}\right\}$, and all $(s, t)$

$$
\begin{aligned}
\int_{0}^{t} \exp \left(\int_{s}^{t} v_{w}(r) d r\right) V(s) M_{z, i}(s) z_{0} d s & \\
& \geq \int_{0}^{t} \gamma_{r_{2}}(s, t) z_{0} d s .
\end{aligned}
$$

Consequently, for all $z_{0}$, it exists $r_{1}$ and $r_{2}$ such that for all time function $z(t)$ satisfying (17), then for all $t$

$$
\begin{aligned}
& \int_{0}^{t} \gamma_{r_{2}}(s, t) z_{0} d s \\
& \leq \int_{0}^{t} \exp \left(\int_{s}^{t} v_{w}(r) d r\right) V(s) z(s) d s \\
& \leq \int_{0}^{t} \gamma_{r_{1}}(s, t) z_{0} d s .
\end{aligned}
$$

This gives,

$$
\begin{aligned}
& \int_{0}^{t} \exp \left(\int_{s}^{t} v_{w}(r) d r\right) V(s) z(s) d s \\
& \qquad \operatorname{Conv}_{1 \leq r \leq 4^{n} z}\left\{\int_{0}^{t} \gamma_{r}(s, t) z_{0}\right\}
\end{aligned}
$$

Finally, the result with the set of matrix functions $t \mapsto$ $M_{w, i}(t)$ is obtained and defined as the $2 * 4^{n_{z}} * \ell_{z}$ matrix functions of the form :

$$
\begin{array}{r}
M_{w, i}(t) \in\left\{\left[\begin{array}{cc}
\exp \left(t v_{w, \max }\right) & \int_{0}^{t} \gamma_{r}(s, t) \\
0 & M_{z, k}(t)
\end{array}\right],\right. \\
\\
\left.\quad\left[\begin{array}{cc}
\exp \left(t v_{w, \min }\right) & \int_{0}^{t} \gamma_{r}(s, t) \\
0 & M_{z, k}(t)
\end{array}\right]\right\}
\end{array}
$$

with $k$ in $\left\{1, \ldots, \ell_{z}\right\}$ and $r$ in $\left\{1, \ldots, 4^{n_{z}}\right\}$. Note that it leads to

$$
\begin{aligned}
\frac{d M_{w, j}}{d t}(0) \in\left\{\left[\begin{array}{cc}
v_{w, \max } & \gamma_{r}(0,0) \\
0 & \frac{M_{z, k}}{d t}(0)
\end{array}\right],\right. & {\left.\left[\begin{array}{cc}
v_{w, \min } & \gamma_{r}(0,0) \\
0 & \frac{d M_{z, k}}{d t}(0)
\end{array}\right]\right\} }
\end{aligned}
$$

with $k$ in $\left\{1, \ldots, \ell_{z}\right\}$ and $r$ in $\left\{1, \ldots, 4^{n_{z}}\right\}$. With the definition of the functions $\gamma_{r},(18)$ is satisfied. This concludes the proof. 